\title{
Insulin Increases Central Aortic Stiffness in \\ Response To Hyperglycemia in Healthy Humans: A Randomized Four-Way Crossover Study
}

\section{William B Horton ( $\square$ WBH2N@virginia.edu )}

University of Virginia School of Medicine https://orcid.org/0000-0001-8081-1214

Linda A Jahn

University of Virginia

Lee M Hartline

University of Virginia

Kevin W Aylor

University of Virginia

James T Patrie

University of Virginia School of Medicine

Eugene J Barrett

University of Virginia School of Medicine

\section{Research Article}

Keywords: Insulin, Hyperglycemia, Octreotide, Vascular stiffness

Posted Date: March 24th, 2021

DOI: https://doi.org/10.21203/rs.3.rs-72339/v5

License: (c) (i) This work is licensed under a Creative Commons Attribution 4.0 International License.

Read Full License 


\section{Abstract}

Introduction: Increasing arterial stiffness is a feature of vascular aging that is accelerated by conditions that enhance cardiovascular risk, including diabetes mellitus. Multiple studies demonstrate divergence of carotid-femoral pulse wave velocity and augmentation index in persons with diabetes mellitus, though mechanisms responsible for this are unclear.

Materials and Methods: We tested the effect of acutely and independently increasing plasma glucose, plasma insulin, or both on hemodynamic function and markers of arterial stiffness (including carotidfemoral pulse wave velocity, augmentation index, forward and backward wave reflection amplitude, and wave reflection magnitude) in a four-arm, randomized study of healthy young adults.

Results: Carotid-femoral pulse wave velocity increased only during hyperglycemic-hyperinsulinemia $(+0.36 \mathrm{~m} / \mathrm{s} ; \mathrm{p}=0.032)$, while other markers of arterial stiffness did not change (all $p>0.05)$. Heart rate (+3.62 bpm; $p=0.009)$, mean arterial pressure $(+4.14 \mathrm{mmHg} ; \mathrm{p}=0.033)$, central diastolic blood pressure $(+4.16 \mathrm{mmHg} ; \mathrm{p}=0.038)$, and peripheral diastolic blood pressure $(+4.09 \mathrm{mmHg} ; \mathrm{p}=0.044)$ also significantly increased during hyperglycemic-hyperinsulinemia.

Conclusions: Hyperglycemic-hyperinsulinemia acutely increased cfPWV, heart rate, mean arterial pressure, and diastolic blood pressure in healthy humans, perhaps reflecting enhanced sympathetic tone. Whether repeated bouts of hyperglycemia with hyperinsulinemia contribute to chronically-enhanced arterial stiffness remains unknown. (ClinicalTrials.gov number NCT03520569; registered 9 May 2018).

Clinical Trial Information: ClinicalTrials.gov identifier NCT03520569 (registered 9 May 2018).

\section{Introduction}

Arterial stiffness develops from dynamic interactions between structural and cellular elements of the vessel wall influenced by both hemodynamic forces and extrinsic factors (including hormones, salt, and glucose $)^{1}$. Arterial stiffness increases with vascular aging due to gradual loss of arterial elasticity ${ }^{2}$ and is accelerated by conditions that increase cardiovascular risk, including diabetes mellitus (DM) ${ }^{3}$. Notably, arterial stiffness often antedates and is itself a strong risk factor for many cardiovascular diseases (e.g., heart failure, myocardial infarction, etc. $)^{3,4}$. Data from numerous studies demonstrate that increased arterial stiffness (assessed by carotid-femoral pulse wave velocity (cfPWV) ${ }^{5-11}$, augmentation index $(\mathrm{Alx})^{12,13}$, reflection magnitude $(\mathrm{RM})^{14,15}$, or backward wave amplitude $\left.(\mathrm{Pb})^{16}\right)$ predicts cardiovascular 
events and/or mortality and may explain some of the notable residual cardiovascular risk associated with even well-controlled hypertension ${ }^{17}$.

Arterial stiffness increases with deteriorating glucose tolerance status ${ }^{18}$ and is associated with insulin resistance ${ }^{19}$; however, the independent and combined roles of acute hyperglycemia and/or hyperinsulinemia on arterial stiffness are not well-defined ${ }^{20}$. Moreover, prior studies have identified divergence of arterial stiffness markers (specifically increased cfPWV and reduced Alx) in persons with $\mathrm{DM}^{21-23}$, though mechanisms responsible for this are also unclear. A recent editorial by Currie and Delles encouraged investigation of healthy cohorts to better-understand mechanisms contributing to alterations in arterial stiffness ${ }^{24}$. As arterial stiffness is increased in even adolescents with $\mathrm{DM}^{25}$, healthy young adults without vascular dysfunction comprise the ideal population in which to investigate metabolic mechanisms linked to acute changes in arterial stiffness. With these points in mind, we sought to quantify the independent effects of elevated concentrations of insulin, glucose, and both on arterial stiffness in healthy humans. Greenfield et al. previously showed that Alx decreases in response to the magnitude of postprandial glucose and insulin responses after low- and high-carbohydrate meal ingestion ${ }^{26}$. To isolate the effects of insulin and glucose from those of incretins and the autonomic changes that occur with oral glucose or meal ingestion, we used intravenous glucose and insulin infusions with co-administration of octreotide (OCT) to block endogenous insulin secretion. We then measured cfPWV, Alx, RM, forward wave amplitude (Pf), $\mathrm{Pb}$, and systemic hemodynamic function during euglycemia, hyperglycemia, euglycemic-hyperinsulinemia, and hyperglycemic-hyperinsulinemia.

\section{Materials And Methods}

\section{Recruitment and Study Population}

Recruitment for this study was achieved by public advertisement. Healthy young adults met inclusion criteria if they were $\geq 18$ and $\leq 35$ years old, had normal body mass index $\left(18-25 \mathrm{~kg} / \mathrm{m}^{2}\right)$, and had a screening fasting plasma glucose $<100 \mathrm{mg} / \mathrm{dL}$ and a screening blood pressure $<140 / 90 \mathrm{mmHg}$. Subjects were excluded if they were current smokers or quit smoking $<5$ years ago, had a first-degree relative with type $2 \mathrm{DM}$, were taking vasoactive medications (e.g., anti-hypertensives, diuretics, statins, etc.), were pregnant (i.e., positive pregnancy test) or nursing, had history of allergy or prior adverse reaction to octreotide, or significant morbidity that could, in the investigator's opinion, affect outcome measures or subject safety.

\section{Clinical Assessment and Initial Screening}

All screening visits and infusion studies were conducted at the University of Virginia (UVA) Clinical Research Unit (CRU). Each subject gave written informed consent at their initial visit prior to being carefully screened to verify inclusion/exclusion criteria. Screening included a detailed medical history and physical examination along with fasting measures of complete blood count, comprehensive metabolic panel, lipid panel, plasma glucose, and serum pregnancy test. 


\section{Study Design}

We followed the Consolidated Standards of Reporting Trials (CONSORT) guidelines ${ }^{27}$ to analyze and report this randomized trial. Randomization of study sequence was conducted by study personnel using a 1:1:1:1 starting allocation from a computer-generated sequence program ${ }^{28}$. The protocol was designed to assess: (1) Protocol A followed by Protocol B (or vice-versa), with subsequent crossover to Protocols $C$ and D (in randomized order); or (2) Protocol C followed by Protocol D (or vice-versa), with subsequent crossover to Protocols A and B (in randomized order). Subjects underwent four infusion protocols (Figure 1) to test the discrete effects of euglycemia, hyperglycemia, euglycemic-hyperinsulinemia, and hyperglycemic-hyperinsulinemia on arterial stiffness. All protocols were approved by the UVA Institutional Review Board (\#19948), with each protocol being performed $\geq 2$ but $\leq 4$ weeks apart for individual subjects to allow a washout period between studies. Within each protocol, we measured cfPWV, Alx, Pf, $\mathrm{Pb}, \mathrm{RM}$, systolic blood pressure, diastolic blood pressure, pulse pressure, mean arterial pressure, and heart rate immediately before (i.e., baseline) and at the end of the infusion period (Figure 1). All vascular assessments for this study were measured per expert recommendations by the same trained operator ${ }^{29}$. After randomization, study personnel were blinded to subject and protocol when evaluating outcome measures. Study participants were instructed to avoid alcohol, exercise, and caffeine for 24 hours and to fast overnight prior to admission to the CRU. We placed intravenous catheters in the right wrist for blood sampling and in the right antecubital fossa for administration of insulin, glucose, and octreotide (OCT). Studies then began with simultaneous infusion of regular insulin and OCT to maintain plasma insulin near basal levels. We did not replace glucagon or growth hormone, as there is currently no evidence that acutely suppressing basal levels of either hormone affects vascular function.

Protocol A (Euglycemia): A 90-minute saline infusion was initiated, with baseline vascular function measurements obtained during the final 30 minutes (Figure 1A). Then, OCT (30 ng/kg/min) with basal insulin replacement $(0.15 \mathrm{mU} / \mathrm{min} / \mathrm{kg})$ was infused for 240 minutes. Blood glucose was sampled every 10 minutes and plasma insulin every 30 minutes. Euglycemia (EU) was maintained by a variable-rate glucose infusion using the negative feedback principle ${ }^{30}$. We repeated vascular measurements over the final 30 minutes of OCT infusion.

Protocol B (Isolated Hyperglycemia): Octreotide with basal insulin replacement was continuously infused for 90 minutes while euglycemia was maintained. Baseline vascular measurements were obtained over the final 30 minutes (Figure 1B). Then, a primed, variable-rate $20 \%$ dextrose infusion began to acutely raise and subsequently maintain blood glucose at $\sim 200 \mathrm{mg} / \mathrm{dL}$ using the hyperglycemic clamp method $^{30}$. Blood glucose was sampled every 5 minutes and plasma insulin every 30 minutes, with repeat vascular measurements obtained over the final 30 minutes of hyperglycemia.

Protocol C (Euglycemic-Hyperinsulinemia): Euglycemia was maintained throughout this protocol by a variable-rate $20 \%$ dextrose infusion using the negative feedback principle ${ }^{30}$. Baseline arterial stiffness measurements were obtained during the final 30 minutes of an OCT (30 ng/kg/min) plus basal insulin $(0.15 \mathrm{mU} / \mathrm{min} / \mathrm{kg})$ infusion (Figure 1C). Then, hyperinsulinemia was initiated with a primed $(2 \mathrm{mU} / \mathrm{kg} / \mathrm{min}$ 
x $10 \mathrm{~min})$, continuous ( $1 \mathrm{mU} / \mathrm{kg} / \mathrm{min} \times 110 \mathrm{~min}$ ) infusion and OCT continued for 120 minutes. Blood glucose was sampled every 5 minutes and plasma insulin every 30 minutes, with repeat arterial stiffness and hemodynamic measurements obtained during the final 30 minutes of the insulin clamp.

Protocol D (Hyperglycemic-Hyperinsulinemia): As in Protocol C, a variable-rate $20 \%$ dextrose infusion maintained euglycemia while OCT (30 ng/ $\mathrm{kg} / \mathrm{min})$ and basal insulin $(0.15 \mathrm{mU} / \mathrm{min} / \mathrm{kg}$ ) were simultaneously infused for the first 90 minutes of this study (Figure 1D). Then, a primed, variable-rate $20 \%$ dextrose infusion began to acutely raise and subsequently maintain blood glucose at $\sim 200 \mathrm{mg} / \mathrm{dL}$. Blood glucose was then sampled every 5 minutes and plasma insulin every 30 minutes, with baseline arterial stiffness measurements obtained over the final 30 minutes of the 120-minute hyperglycemic period (Figure 1B). Subsequently, hyperinsulinemia was initiated with a primed ( $2 \mathrm{mU} / \mathrm{kg} / \mathrm{min} \times 10 \mathrm{~min}$ ), continuous ( $1 \mathrm{mU} / \mathrm{kg} / \mathrm{min} \times 110 \mathrm{~min})$ infusion with OCT and hyperglycemia maintained for 120 minutes. Blood glucose was sampled every 5 minutes with plasma insulin every 30 minutes, and repeat arterial stiffness and hemodynamic measurements were again obtained during the final 30 minutes of the insulin clamp.

Hemodynamic Function: Clinical hemodynamic assessments were obtained at two time points during each protocol (Figure 1). Blood pressure, pulse pressure, mean arterial pressure, and heart rate were measured and/or calculated with a SphygmoCor® tonometer (ATCOR USA; Napierville, IL).

Carotid-Femoral Pulse Wave Velocity (cfPWV): To assess central aortic stiffness, cfPWV was measured with a SphygmoCor tonometer. To minimize the effects of sympathetic activity on cfPWV measurements, participants laid supine in a temperature-controlled room for at least 15 minutes prior to measurement. We measured the distance from the suprasternal notch to the carotid pulse and from the suprasternal notch to the femoral pulse on the same side. For each cfPWV measure, 10 seconds of carotid and 10 seconds of femoral arterial waveforms were recorded. cfPWV measures were made in duplicate and the mean value was reported. cfPWV intraobserver reliability was also assessed by having the operator record three serial cfPWV measurements on the same subject over a 4-hour period. The coefficient of variation for cfPWV was $3.63 \%$, indicating good intraobserver reliability ${ }^{31}$. Of note, the cfPWV data in this manuscript were included in a separate report examining macro- and microvascular functional responses to the two insulin clamp protocols ${ }^{32}$.

Augmentation Index (Alx): To assess muscular conduit arterial stiffness, we measured Alx noninvasively with a SphygmoCor tonometer. Alx measurements were obtained at the radial artery by the same trained operator with participants lying in the supine position in a temperature-controlled room for at least 15 minutes prior to measurement. Alx was calculated as the difference of the amplitude of the late systolic peak to the early systolic peak divided by the pulse pressure and expressed as a percentage. Alx values were determined for each pulse over a 30 second period, and a mean value was calculated by the device for each patient and corrected for a heart rate of 75 beats per minute. 
Wave Separation Analyses (Pf, Pb, and RM): We derived the central aortic pressure waveform by recording radial artery pressure waves through aplanation tonometry using a SphygmoCor tonometer. Briefly, after 20 sequential waveforms were acquired and an ensemble was averaged, a validated general transfer function was used to synthesize the central aortic pressure wave noninvasively. Wave separation analysis then decomposed pressure and flow waveforms into their forward (Pf) and backward (Pb) components ${ }^{29}$. Reflection magnitude (RM) was calculated as the ratio of the amplitudes of the backward/forward pressure waves ${ }^{33}$.

\section{Biochemical Analyses}

Complete blood count, comprehensive metabolic panel, lipid panel, fasting plasma glucose, and serum pregnancy tests were assayed at the UVA Clinical Chemistry Laboratory. Plasma glucose was measured with the YSI 2700 Biochemistry Analyzer (Yellow Springs Instrument Company; Yellow Springs, $\mathrm{OH}$ ). Plasma insulin was measured with the ALPCO Insulin ELISA (ALPCO; Salem, NH). Insulin assays were read on a Synergy 2 microplate reader (BioTek; Winooski, VT).

\section{Data Storage}

Study data are stored in a Research Electronic Data Capture (REDCap) ${ }^{34}$ project file repository hosted at UVA. The datasets generated and/or analyzed during the current study are available from the corresponding author upon reasonable request.

\section{Statistical Analyses}

Sample Size: A sample size calculation using the Cohen's $d$ effect size from a previous study of cfPWV changes during euglycemic-hyperinsulinemia ${ }^{35}$ in metabolic syndrome subjects indicated that $\geq 8$ participants in each protocol would have $\geq 80 \%$ power to detect meaningful cfPWV changes in the current study.

Outcomes: The primary outcome for each protocol was change in cfPWV. Secondary outcomes for each protocol included changes in $\mathrm{Al}, \mathrm{Pf}, \mathrm{Pb}, \mathrm{RM}$, systolic blood pressure, diastolic blood pressure, mean arterial pressure, pulse pressure, and plasma insulin.

Descriptive Summarization: Patient demographics were summarized using common descriptive statistics. The arithmetic mean and standard error of mean, standard deviation, median, and interquartile range were used to summarize continuous scaled outcome measures.

Data Transformation and Summarization: Given that the data distributions for some outcome measures were initially skewed, we rescaled the pre- and post-intervention outcome measures for each protocol to the natural logarithmic scale (i.e., $\log _{\mathrm{e}}$ ). The analytical outcome data were then derived by subtracting the $\log _{\mathrm{e}}$ transformed pre-intervention outcome measures from the $\log _{\mathrm{e}}$ transformed post-intervention outcome variable measures. For all outcome measures, the point estimate for the mean pre-intervention 
outcome measures, the point estimate for the mean pre- to post-intervention outcome measure change, and the point estimate for the difference between the mean of the pre-intervention outcome measures and the mean pre- to post-intervention outcome measure change for each protocol were converted via natural logarithmic antilog transformation (i.e., $\mathrm{e}^{\mathrm{x}}$ ) to a geometric mean ratio scale.

Outcome Measure Analyses: The pre- to post-intervention outcome variable change for each protocol was compared by linear mixed model (LMM) analysis of covariance. Significance was set at $a=0.05$ (twotailed test). All statistical analyses were performed with SAS Studio 3.8 (SAS; Cary, NC). LMM Specification: The analytical outcome data of each protocol served as the dependent variable measurements of the LMM. An indicator variable to identify the protocol (i.e., A, B, C, or D) served as one of the LMM independent variables, and the $\log _{e}$ transformed pre-intervention outcome measurements of each protocol served as a second LMM independent variable. Note that the $\log _{\mathrm{e}}$ transformed preintervention measurements were included as part of the LMM so that the between-admission comparison of the mean pre- to post-intervention change in the outcome measure could be standardized to a common reference pre-intervention measurement value. Hypothesis testing: Primary hypotheses under the null tested whether the mean within-protocol change in each outcome measure was equal to zero.

\section{Results}

\section{Baseline Subject Characteristics and Demographics}

Table 1 details baseline demographics of the 19 total study participants. All had normal BMI, blood pressure, and fasting plasma glucose. Notably, 13 subjects completed Protocol A, 10 subjects Protocol B, 14 subjects Protocol C, and 12 subjects Protocol D. Nine subjects completed all four protocols. Three subjects voluntarily withdrew after completing only Protocols $C$ and $D$, two after completing only Protocol $\mathrm{C}$, and two after completing only Protocol A. One subject moved out of the area after completing Protocols $A$ and $B$, one experienced scheduling difficulty after completing only Protocols $A$ and $D$, and one completed Protocol $\mathrm{D}$ but then experienced abdominal cramping (due to octreotide) that led to study termination during their second admission. An electrode wire malfunction resulted in no post-intervention assessment of cfPWV for one subject in Protocol B.

\section{Plasma Insulin and Glucose Concentrations}

Figure 2 shows the time course for mean plasma glucose (upper panel), mean glucose infusion rate (middle panel), and mean plasma insulin levels (lower panel) throughout each protocol. Plasma glucose levels rose significantly from baseline within Protocols $B$ and D, and plasma insulin concentrations rose significantly from baseline within Protocols $C$ and $D$. These increases did not differ between respective protocol pairs.

\section{Arterial Stiffness}


Table 2 shows the summary statistics for pre- and post-intervention measures of arterial stiffness within each protocol. cfPWV did not change during euglycemia, hyperglycemia, or euglycemic-hyperinsulinemia (all $p>0.05)$, but significantly increased after hyperinsulinemia was added to hyperglycemia $(+0.36 \mathrm{~m} / \mathrm{s}$; $p=0.032$ ). Alx significantly decreased during euglycemia (Protocol $A$ ), but did not significantly change within any other protocol. There were no significant changes in Pf, Pb, or RM within any protocol.

\section{Hemodynamic Function}

Table 3 details the within-protocol changes for all hemodynamic parameters. Heart rate $(+3.62 \mathrm{bpm}$; $\mathrm{p}=0.009)$, mean arterial pressure $(+4.14 \mathrm{mmHg} ; \mathrm{p}=0.033)$, central diastolic blood pressure $(+4.16 \mathrm{mmHg}$; $p=0.038)$, and peripheral diastolic blood pressure $(+4.09 \mathrm{mmHg} ; \mathrm{p}=0.044)$ significantly increased during only hyperglycemic-hyperinsulinemia. There were no significant changes in aortic or peripheral pulse pressure or systolic blood pressure within any protocol (all $p>0.05$ ).

\section{Discussion}

To our knowledge, this study is the first to investigate the discrete effects of acute hyperglycemia and hyperinsulinemia on cfPWV, Alx, RM, Pf, and $\mathrm{Pb}$ in the same subjects, with several novel observations warranting discussion. First, the combination of hyperglycemia and hyperinsulinemia increased cfPWV, while isolated hyperglycemia or hyperinsulinemia did not. Second, hyperglycemic-hyperinsulinemia acutely increased heart rate, mean arterial pressure, and diastolic blood pressure. Finally, no significant changes were seen in Alx, RM, Pf, and Pb during hyperglycemic-hyperinsulinemia.

Prior work from Puzantian et al. found that acute hyperglycemia (using pancreatic clamping methodology in healthy subjects) did not alter cfPWV, but they noted that further studies were needed to determine the independent and combined roles of glucose and insulin on cfPWV ${ }^{20}$. To our knowledge, the current study is the first to investigate this question. Infusion of OCT allowed us to isolate the effects of insulin and glucose and provide the first evidence that moderate hyperglycemia unmasks an action of physiologic hyperinsulinemia to increase central aortic stiffness in healthy humans. Here we must note that heart rate, mean arterial pressure, and diastolic blood pressure also rose significantly during hyperglycemic-hyperinsulinemia. A positive association between heart rate and cfPWV has been demonstrated in recent studies ${ }^{36,37}$. Bikia et al. recently performed an in-silico evaluation of the impact of heart rate on cfPWV and confirmed that small heart rate changes only slightly alter cfPWV, but they suggested that a more clinically significant impact on cfPWV should be considered in cases wherein heart rate might vary to a greater extent ${ }^{38}$. The mean increase in heart rate and cfPWV during hyperglycemic-hyperinsulinemia in the current study were $3.62 \mathrm{bpm}$ and $0.4 \mathrm{~m} / \mathrm{s}$, respectively. Prior reports show that the effect of heart rate on cfPWV is small and on the order of $0.02 \mathrm{~m} / \mathrm{s}$ per $1 \mathrm{bpm}$ change 36,37 , thus increased heart rate alone is unlikely to explain the increase in cfPWV observed during hyperglycemic-hyperinsulinemia. We also note that acute hyperglycemic-hyperinsulinemia significantly increased mean arterial pressure and diastolic blood pressure without altering systolic blood pressure. These findings mirror prior reports demonstrating that increases in heart rate ${ }^{39-41}$ and arterial stiffness ${ }^{42}$, 
${ }^{43}$ precede the development of systolic hypertension. Similarly, acute stiffening of the thoracic aorta and its branches (i.e., the arterial distribution represented by cfPWV) corresponds to increases in mean arterial pressure ${ }^{44}$. Increased transmural pressure at the level of the central aorta causes the elastic lamellae of this large artery to stretch and become stiffer, resulting in higher cfPWV ${ }^{44}$. The increase of mean arterial pressure observed in the current study corresponded to an acute increase in cfPWV. Such increases of vascular tone, occurring repetitively, may contribute to vascular remodeling ${ }^{45}$. Further studies are needed to investigate and test this hypothesis.

We also found that Alx, RM, Pf, and Pb did not significantly change during hyperglycemichyperinsulinemia. This result aligns with previous studies reporting that increased cfPWV is not associated with elevated Alx in persons with $\mathrm{DM}^{21-23}$. Our prior work has also shown that insulin has opposing effects on cfPWV and Alx in various metabolic conditions. Specifically, insulin (during euglycemia) acutely reduced Alx in both healthy and metabolic syndrome subjects, but increased cfPWV in metabolic syndrome subjects only ${ }^{35}$. In that study, metabolic syndrome subjects were insulin-resistant and had chronically higher fasting plasma glucose and insulin concentrations (i.e., the milieu of metabolic syndrome and type 2 DM). Lacy et al. hypothesized that dissipation of the energy of the incident pressure wave in persons with DM blunting wave reflections might explain this phenomenon ${ }^{21}$. In other words, it is reduced wave reflections, not the increased outgoing pressure wave, that result in the attenuation of Alx in persons with $\mathrm{DM}^{21,22}$. In contrast, Khoshdel et al. theorized that Alx underestimates arterial stiffness in persons with DM because of their wider pulse pressure compared to healthy controls $^{23}$. In this hypothesis, the main determinant of Alx is pulse pressure and not the reflected wave ${ }^{22}$. The current study provides some clarification for this debate. In our healthy subjects with intact vascular function, the acute combination of hyperglycemia with hyperinsulinemia increased heart rate, mean arterial pressure, diastolic blood pressure, and cfPWV without significantly altering pulse pressure. We note that cfPWV is a direct measure of arterial stiffness, while Alx and pulse pressure are surrogate indicators of arterial stiffness ${ }^{46}$. Pulse pressure did not significantly change within the hyperglycemichyperinsulinemia protocol (or within any protocol) of the current study. These results suggest blunted wave reflections may better explain why Alx likely underestimates arterial stiffness in type $2 \mathrm{DM}^{22}$, and that the acute changes in cfPWV are independent of pulse pressure.

Taken together, our findings suggest a potential mechanism whereby acute, repetitive metabolic alterations could impact hemodynamic function and aortic stiffness. These results may have implications for the accelerated vascular aging seen in persons with both types 1 and 2 DM who regularly experience the acute combination of hyperglycemia and hyperinsulinemia. We caution, however, that the chronic impact of glucose and insulin per se on arterial stiffness includes a more complex vascular remodeling not seen with the acute metabolic interventions studied here. For example, widened pulse pressure has been associated with DM in many studies ${ }^{47-49}$, but we did not see a change in pulse pressure within any protocol of the current study. Acute alterations in arterial stiffness result from changes in endothelial function, vascular tone, heart rate, and other causes, whereas chronic arterial 
stiffening is mainly due to vascular remodeling, fibrosis, and calcification ${ }^{50}$. Whether repeated, acute measurable episodes of arterial stiffening contribute to long-term remodeling, fibrosis, and vascular injury remains a point of speculation. Recent work has shown that hyperglycemic-hyperinsulinemia significantly increases circulating norepinephrine (but not epinephrine) in healthy humans ${ }^{51}$. The increases in cfPWV, heart rate, mean arterial pressure, and diastolic blood pressure observed during hyperglycemic-hyperinsulinemia in the current study mimic the enhanced sympathetic nervous system activity seen in insulin-resistant states like obesity and type $2 \mathrm{DM}^{52,53}$. Our results, combined with these prior reports, point to sympathetic stimulation as a potential explanation for the hemodynamic changes seen during acute hyperglycemic-hyperinsulinemia. Unfortunately, we did not measure sympathetic nervous system activity in our study. Future work will focus on investigating this question and establishing a mechanistic basis for the hemodynamic effects observed during hyperglycemichyperinsulinemia.

While the current study provides novel insight into acute vascular changes during hyperglycemichyperinsulinemia, several study limitations should be mentioned. First, we studied a small number of healthy young adults and the study was powered to detect within-protocol responses to glucose and insulin. Thus, we did not examine between-protocol differences. Second, persons with DM or those who are older and/or less healthy might respond differently to our study protocols. Third, while Alx is widely

used to evaluate arterial stiffness, conflicting data exist in regards to its validity ${ }^{54,55}$. A recent scientific statement from the American Heart Association recommended using wave separation analysis, as opposed to aortic Alx alone, when investigations are focused on the role of wave reflection ${ }^{29}$. These considerations led us to examine Pf, $\mathrm{Pb}$, and $\mathrm{RM}$ in the current study. Fourth, mean cfPWV baseline values varied between protocols, though these differences were not statistically significant. This raises the issue of whether the significant within-protocol change seen during hyperglycemic-hyperinsulinemia was influenced by some "regression to the mean." We considered this, but the concomitant changes seen in heart rate, diastolic blood pressure, and mean arterial pressure rather suggest the cfPWV change is legitimate and possibly due to the sympathetic mechanisms discussed above. Finally, we cannot rule out that OCT has in some unknown manner skewed the observed vascular responses, and this possibility should not be discounted. We do note, however, that no vasoactive effects have been identified in previous studies using a similar dose of $\mathrm{OCT}^{51,56-58}$ and that OCT infusion does not alter the hemodynamic effects of acute hyperglycemia ${ }^{59}$.

\section{Conclusion}

Hyperglycemic-hyperinsulinemia acutely increased cfPWV, heart rate, mean arterial pressure, and diastolic blood pressure in healthy humans, perhaps reflecting enhanced sympathetic tone. Whether repeated bouts of hyperglycemia with hyperinsulinemia (as occur regularly during the postprandial period in persons with DM and insulin-resistant syndromes) contribute to chronically-enhanced arterial stiffness remains unknown. 


\section{Abbreviations}

cfPWV = carotid-femoral pulse wave velocity; $\mathrm{DM}=$ diabetes mellitus; $\mathrm{OCT}=$ octreotide; $\mathrm{Al}=$ augmentation index; $\mathrm{Pb}=$ backward wave amplitude; $\mathrm{RM}=$ reflection magnitude; $\mathrm{Pf}=$ forward wave amplitude; UVA= University of Virginia; $\mathrm{CRU}=$ clinical research unit.

\section{Declarations}

Ethics Approval and Consent to Participate: All protocols were approved by the University of Virginia Institutional Review Board (\#19948). Each subject gave written informed consent at their initial screening visit prior to study participation.

Availability of Data and Materials: The datasets used and/or analyzed during the current study are available from the corresponding author on reasonable request.

Declaration of Conflicting Interests: The authors declare that they have no conflicts of interest to disclose.

Funding: This work was supported in part by National Institutes of Health research grants F32HL14230401, KL2TR003016, and UL1TR003015 (to WBH), and R01DK073059 and R01HL142250 (to EJB).

\section{References}

1. Zieman SJ, Melenovsky V and Kass DA. Mechanisms, pathophysiology, and therapy of arterial stiffness. Arterioscler Thromb Vasc Biol 2005; 25: 932-943. 2005/02/26. DOI: 10.1161/01.ATV.0000160548.78317.29.

2. Bolton E and Rajkumar C. The ageing cardiovascular system. Reviews in Clinical Gerontology 2011; 21: 99-109. DOI: 10.1017/s0959259810000389.

3. Lyle AN and Raaz U. Killing Me Unsoftly: Causes and Mechanisms of Arterial Stiffness. Arterioscler Thromb Vasc Biol 2017; 37: e1-e11. 2017/01/27. DOI: 10.1161/ATVBAHA.116.308563.

4. Yu S and McEniery CM. Central Versus Peripheral Artery Stiffening and Cardiovascular Risk. Arterioscler Thromb Vasc Biol 2020; 40: 1028-1033. 2020/03/20. DOI: 10.1161/ATVBAHA.120.313128.

5. Boutouyrie $\mathrm{P}$, Tropeano Al, Asmar R, et al. Aortic stiffness is an independent predictor of primary coronary events in hypertensive patients: a longitudinal study. Hypertension 2002; 39: 10-15. 2002/01/19. DOI: 10.1161/hy0102.099031.

6. Laurent S, Boutouyrie P, Asmar R, et al. Aortic stiffness is an independent predictor of all-cause and cardiovascular mortality in hypertensive patients. Hypertension 2001; 37: 1236-1241. 2001/05/23. DOI: 10.1161/01.hyp.37.5.1236.

7. Ben-Shlomo Y, Spears M, Boustred C, et al. Aortic pulse wave velocity improves cardiovascular event prediction: an individual participant meta-analysis of prospective observational data from 17,635 
subjects. J Am Coll Cardiol 2014; 63: 636-646. 2013/11/19. DOI: 10.1016/j.jacc.2013.09.063.

8. London GM, Blacher J, Pannier B, et al. Arterial wave reflections and survival in end-stage renal failure. Hypertension 2001; 38: 434-438. 2001/09/22. DOI: 10.1161/01.hyp.38.3.434.

9. Cruickshank K, Riste L, Anderson SG, et al. Aortic pulse-wave velocity and its relationship to mortality in diabetes and glucose intolerance: an integrated index of vascular function? Circulation 2002; 106 : 2085-2090. 2002/10/16. DOI: 10.1161/01.cir.0000033824.02722.f7.

10. Sutton-Tyrrell K, Najjar SS, Boudreau RM, et al. Elevated aortic pulse wave velocity, a marker of arterial stiffness, predicts cardiovascular events in well-functioning older adults. Circulation 2005; 111: 3384-3390. 2005/06/22. DOI: 10.1161/CIRCULATIONAHA.104.483628.

11. Mitchell GF, Hwang SJ, Vasan RS, et al. Arterial stiffness and cardiovascular events: the Framingham Heart Study. Circulation 2010; 121: 505-511. 2010/01/20. DOI:

10.1161/CIRCULATIONAHA.109.886655.

12. Nurnberger J, Keflioglu-Scheiber A, Opazo Saez AM, et al. Augmentation index is associated with cardiovascular risk. J Hypertens 2002; 20: 2407-2414. 2002/12/11. DOI: 10.1097/00004872200212000-00020.

13. Tynjala A, Forsblom C, Harjutsalo V, et al. Arterial Stiffness Predicts Mortality in Individuals With Type 1 Diabetes. Diabetes Care 2020; 43: 2266-2271. 2020/07/11. DOI: 10.2337/dc20-0078.

14. Chirinos JA, Kips JG, Jacobs DR, Jr., et al. Arterial wave reflections and incident cardiovascular events and heart failure: MESA (Multiethnic Study of Atherosclerosis). J Am Coll Cardiol 2012; 60: 2170-2177. 2012/10/30. DOI: 10.1016/j.jacc.2012.07.054.

15. Zamani P, Jacobs DR, Jr., Segers P, et al. Reflection magnitude as a predictor of mortality: the MultiEthnic Study of Atherosclerosis. Hypertension 2014; 64: 958-964. 2014/09/27. DOI: 10.1161/HYPERTENSIONAHA.114.03855.

16. Wang KL, Cheng HM, Sung SH, et al. Wave reflection and arterial stiffness in the prediction of 15-year all-cause and cardiovascular mortalities: a community-based study. Hypertension 2010; 55: 799-805. 2010/01/13. DOI: 10.1161/HYPERTENSIONAHA.109.139964.

17. Niiranen TJ, Kalesan B, Hamburg NM, et al. Relative Contributions of Arterial Stiffness and Hypertension to Cardiovascular Disease: The Framingham Heart Study. J Am Heart Assoc 2016; 5 2016/12/03. DOI: 10.1161/JAHA.116.004271.

18. Henry RM, Kostense PJ, Spijkerman AM, et al. Arterial stiffness increases with deteriorating glucose tolerance status: the Hoorn Study. Circulation 2003; 107: 2089-2095. 2003/04/16. DOI: 10.1161/01.CIR.0000065222.34933.FC.

19. Sengstock DM, Vaitkevicius PV and Supiano MA. Arterial stiffness is related to insulin resistance in nondiabetic hypertensive older adults. J Clin Endocrinol Metab 2005; 90: 2823-2827. 2005/02/25. DOI: $10.1210 /$ jc. $2004-1686$.

20. Puzantian $\mathrm{H}$, Teff $\mathrm{K}$ and Townsend RR. Investigating the effect of glucose on aortic pulse wave velocity using pancreatic clamping methodology. Biol Res Nurs 2015; 17: 270-275. 2015/03/25. DOI: $10.1177 / 1099800415574902$. 
21. Lacy PS, O'Brien DG, Stanley AG, et al. Increased pulse wave velocity is not associated with elevated augmentation index in patients with diabetes. J Hypertens 2004; 22: 1937-1944. 2004/09/14. DOI: 10.1097/00004872-200410000-00016.

22. Zhang M, Bai Y, Ye P, et al. Type 2 diabetes is associated with increased pulse wave velocity measured at different sites of the arterial system but not augmentation index in a Chinese population. Clin Cardiol 2011; 34: 622-627. 2011/10/14. DOI: 10.1002/clc.20956.

23. Khoshdel AR and Carney SL. Increased pulse wave velocity is not associated with elevated augmentation index in patients with diabetes. J Hypertens 2005; 23: 669-670; author reply 670-661. 2005/02/18. DOI: 10.1097/01.hjh.0000160229.24715.9a.

24. Currie G and Delles C. Healthy Vascular Aging. Hypertension 2017; 70: 229-231. 2017/06/01. DOI: 10.1161/HYPERTENSIONAHA.117.09122.

25. Urbina EM, Kimball TR, Khoury PR, et al. Increased arterial stiffness is found in adolescents with obesity or obesity-related type 2 diabetes mellitus. J Hypertens 2010; 28: 1692-1698. 2010/07/22. DOI: 10.1097/HJH.0b013e32833a6132.

26. Greenfield JR, Samaras K, Chisholm DJ, et al. Effect of postprandial insulinemia and insulin resistance on measurement of arterial stiffness (augmentation index). Int J Cardiol 2007; 114: 50-56. 2006/05/06. DOI: 10.1016/j.ijcard.2005.12.007.

27. Dwan K, Li T, Altman DG, et al. CONSORT 2010 statement: extension to randomised crossover trials. BMJ 2019; 366: 14378. 2019/08/02. DOI: 10.1136/bmj.14378.

28. Urbaniak GC, Plous, S. Research Randomizer (Version 4.0), http://www.randomizer.org/ (2013, accessed 06/04/2020).

29. Townsend RR, Wilkinson IB, Schiffrin EL, et al. Recommendations for Improving and Standardizing Vascular Research on Arterial Stiffness: A Scientific Statement From the American Heart Association. Hypertension 2015; 66: 698-722. 2015/07/15. DOI: 10.1161/HYP.0000000000000033.

30. DeFronzo RA, Tobin JD and Andres R. Glucose clamp technique: a method for quantifying insulin secretion and resistance. Am J Physio/ 1979; 237: E214-223. 1979/09/01. DOI:

10.1152/ajpendo.1979.237.3.E214.

31. Shechtman O. The Coefficient of Variation as an Index of Measurement Reliability. In: Doi SAR and Williams GM (eds) Methods of Clinical Epidemiology. Berlin, Heidelberg: Springer Berlin Heidelberg, 2013, pp.39-49.

32. Horton WB, Jahn LA, Hartline LM, et al. Hyperglycemia does not Inhibit Insulin's Effects on Microvascular Perfusion in Healthy Humans: A Randomized Crossover Study. Am J Physiol Endocrinol Metab 2020 2020/08/25. DOI: 10.1152/ajpendo.00300.2020.

33. Westerhof N, Sipkema P, van den Bos GC, et al. Forward and backward waves in the arterial system. Cardiovasc Res 1972; 6: 648-656. 1972/11/01. DOI: 10.1093/cvr/6.6.648.

34. Harris PA, Taylor R, Minor BL, et al. The REDCap consortium: Building an international community of software platform partners. J Biomed Inform 2019; 95: 103208. 2019/05/13. DOI:

10.1016/j.jbi.2019.103208.

Page $13 / 20$ 
35. Jahn LA, Hartline L, Rao N, et al. Insulin Enhances Endothelial Function Throughout the Arterial Tree in Healthy But Not Metabolic Syndrome Subjects. J Clin Endocrinol Metab 2016; 101: 1198-1206. 2016/01/13. DOI: 10.1210/jc.2015-3293.

36. Logan JG and Kim SS. Resting Heart Rate and Aortic Stiffness in Normotensive Adults. Korean Circ J 2016; 46: 834-840. 2016/11/09. DOI: 10.4070/kcj.2016.46.6.834.

37. Tan I, Spronck B, Kiat H, et al. Heart Rate Dependency of Large Artery Stiffness. Hypertension 2016; 68: 236-242. 2016/06/02. DOI: 10.1161/HYPERTENSIONAHA.116.07462.

38. Bikia V, Stergiopulos N, Rovas G, et al. The impact of heart rate on pulse wave velocity: an in-silico evaluation. J Hypertens 2020 2020/08/03. DOI: 10.1097/HJH.0000000000002583.

39. Selby JV, Friedman GD and Quesenberry CP, Jr. Precursors of essential hypertension: pulmonary function, heart rate, uric acid, serum cholesterol, and other serum chemistries. Am J Epidemio/ 1990; 131: 1017-1027. 1990/06/01. DOI: 10.1093/oxfordjournals.aje.a115593.

40. Garrison RJ, Kannel WB, Stokes J, 3rd, et al. Incidence and precursors of hypertension in young adults: the Framingham Offspring Study. Prev Med 1987; 16: 235-251. 1987/03/01. DOI: 10.1016/0091-7435(87)90087-9.

41. Kim JR, Kiefe Cl, Liu K, et al. Heart rate and subsequent blood pressure in young adults: the CARDIA study. Hypertension 1999; 33: 640-646. 1999/02/19. DOI: 10.1161/01.hyp.33.2.640.

42. Kaess BM, Rong J, Larson MG, et al. Aortic stiffness, blood pressure progression, and incident hypertension. JAMA 2012; 308: 875-881. 2012/09/06. DOI: 10.1001/2012.jama.10503.

43. Weisbrod RM, Shiang T, Al Sayah L, et al. Arterial stiffening precedes systolic hypertension in dietinduced obesity. Hypertension 2013; 62: 1105-1110. 2013/09/26. DOI:

10.1161/HYPERTENSIONAHA.113.01744.

44. Franklin SS. Arterial stiffness and hypertension: a two-way street? Hypertension 2005; 45: 349-351. 2005/02/16. DOI: 10.1161/01.HYP.0000157819.31611.87.

45. Chami HA, Vasan RS, Larson MG, et al. The association between sleep-disordered breathing and aortic stiffness in a community cohort. Sleep Med 2016; 19: 69-74. 2016/05/21. DOI: 10.1016/j.sleep.2015.11.009.

46. Lemogoum $D$, Flores $G$, Van den Abeele W, et al. Validity of pulse pressure and augmentation index as surrogate measures of arterial stiffness during beta-adrenergic stimulation. $J$ Hypertens 2004; 22 : 511-517. 2004/04/13. DOI: 10.1097/00004872-200403000-00013.

47. Warren J, Nanayakkara S, Andrianopoulos N, et al. Impact of Pre-Procedural Blood Pressure on LongTerm Outcomes Following Percutaneous Coronary Intervention. J Am Coll Cardiol 2019; 73: 28462855. 2019/06/07. DOI: 10.1016/j.jacc.2019.03.493.

48. Yasuno S, Ueshima K, Oba K, et al. Is pulse pressure a predictor of new-onset diabetes in high-risk hypertensive patients?: a subanalysis of the Candesartan Antihypertensive Survival Evaluation in Japan (CASE-J) trial. Diabetes Care 2010; 33: 1122-1127. 2010/02/27. DOI: 10.2337/dc09-1447.

49. Mule G, Nardi E, Cottone $S$, et al. Relationship of metabolic syndrome with pulse pressure in patients with essential hypertension. Am J Hypertens 2007; 20: 197-203. 2007/01/31. DOI: 
10.1016/j.amjhyper.2006.07.016.

50. Donato AJ, Machin DR and Lesniewski LA. Mechanisms of Dysfunction in the Aging Vasculature and Role in Age-Related Disease. Circ Res 2018; 123: 825-848. 2018/10/26. DOI:

10.1161/CIRCRESAHA.118.312563.

51. Joy NG, Perkins JM, Mikeladze M, et al. Comparative effects of acute hypoglycemia and hyperglycemia on pro-atherothrombotic biomarkers and endothelial function in non-diabetic humans. J Diabetes Complications 2016; 30: 1275-1281. 2016/07/23. DOI:

10.1016/j.jdiacomp.2016.06.030.

52. Moreira MC, Pinto IS, Mourao AA, et al. Does the sympathetic nervous system contribute to the pathophysiology of metabolic syndrome? Front Physio/ 2015; 6: 234. 2015/09/18. DOI: 10.3389/fphys.2015.00234.

53. Esler M, Rumantir M, Wiesner G, et al. Sympathetic nervous system and insulin resistance: from obesity to diabetes. Am J Hypertens 2001; 14: 304S-309S. 2001/11/28. DOI: 10.1016/s08957061(01)02236-1.

54. Zahner GJ, Gruendl MA, Spaulding KA, et al. Association between arterial stiffness and peripheral artery disease as measured by radial artery tonometry. J Vasc Surg 2017; 66: 1518-1526. 2017/08/02. DOI: 10.1016/j.jvs.2017.06.068.

55. Cheng LT, Tang LJ, Cheng L, et al. Limitation of the augmentation index for evaluating arterial stiffness. Hypertens Res 2007; 30: 713-722. 2007/10/06. DOI: 10.1291/hypres.30.713.

56. Beckman JA, Goldfine AB, Gordon MB, et al. Ascorbate restores endothelium-dependent vasodilation impaired by acute hyperglycemia in humans. Circulation 2001; 103: 1618-1623. 2001/03/29. DOI: 10.1161/01.cir.103.12.1618.

57. Beckman JA, Goldfine AB, Gordon MB, et al. Inhibition of protein kinase Cbeta prevents impaired endothelium-dependent vasodilation caused by hyperglycemia in humans. Circ Res 2002; 90: 107111. 2002/01/12. DOI: 10.1161/hh0102.102359.

58. Moller N, Bagger JP, Schmitz O, et al. Somatostatin enhances insulin-stimulated glucose uptake in the perfused human forearm. J Clin Endocrinol Metab 1995; 80: 1789-1793. 1995/06/01. DOI: 10.1210/jcem.80.6.7775624.

59. Marfella R, Nappo F, De Angelis L, et al. Hemodynamic effects of acute hyperglycemia in type 2 diabetic patients. Diabetes Care 2000; 23: 658-663. 2000/06/02. DOI: 10.2337/diacare.23.5.658.

\section{Tables}

Table 1. Baseline Subject Characteristics and Demographics (SEM= standard error of mean). 


\begin{tabular}{c|c|}
\hline Variable & Mean \pm SEM \\
\hline Sex & 12 Female; 7 Male \\
\hline Age (years) & $24.4 \pm 1.0$ \\
\hline Body Mass Index (kg/m²) & $22.4 \pm 0.4$ \\
\hline ystolic Blood Pressure (mmHg) & $114.9 \pm 2.6$ \\
\hline astolic Blood Pressure (mmHg) & $66.5 \pm 1.9$ \\
\hline asting Blood Glucose (mg/dL) & $87.8 \pm 1.4$ \\
\hline Total Cholesterol (mg/dL) & $162.4 \pm 5.4$ \\
\hline LDL Cholesterol (mg/dL) & $92.1 \pm 5.1$ \\
\hline HDL Cholesterol (mg/dL) & $59.9 \pm 2.9$ \\
\hline Triglycerides (mg/dL) & $63.6 \pm 5.0$ \\
\hline
\end{tabular}

Table 2. Summary statistics for pre- and post-intervention cfPWV, AI, Pf, Pb, and RM. (cfPWV=carotid-femoral pulse wave velocity; $\mathrm{AI}=$ augmentation index; $\mathrm{Pf}=$ Forward wave amplitude; $\mathrm{Pb}=$ Backward wave amplitude; $\mathrm{RM}=$ reflection magnitude; $\mathrm{EG}=$ euglycemia; $\mathrm{HG}=$ hyperglycemia; $\mathrm{EH}=$ euglycemic-hyperinsulinemia; $\mathrm{HH}=$ hyperglycemic-hyperinsulinemia; $\mathrm{SD}=\mathrm{standard}$ deviation). 


\begin{tabular}{|c|c|c|c|c|c|}
\hline Vascular Parameter & Protocol & Assessment & $\mathbf{n}$ & Mean \pm SD & P-value \\
\hline \multirow[t]{8}{*}{ cfPWV (m/sec) } & \multirow[t]{2}{*}{ EG } & Pre & 13 & $5.25 \pm 0.47$ & \multirow[t]{2}{*}{0.635} \\
\hline & & Post & 13 & $5.21 \pm 0.64$ & \\
\hline & \multirow[t]{2}{*}{ HG } & Pre & 9 & $4.81 \pm 0.70$ & \multirow[t]{2}{*}{0.830} \\
\hline & & Post & 9 & $4.79 \pm 0.79$ & \\
\hline & \multirow[t]{2}{*}{$\mathrm{EH}$} & Pre & 14 & $5.19 \pm 0.95$ & \multirow[t]{2}{*}{0.495} \\
\hline & & Post & 14 & $5.06 \pm 0.76$ & \\
\hline & \multirow[t]{2}{*}{$\mathrm{HH}$} & Pre & 11 & $4.71 \pm 0.41$ & \multirow[t]{2}{*}{0.032} \\
\hline & & Post & 11 & $5.07 \pm 0.67$ & \\
\hline \multirow[t]{8}{*}{$\mathrm{AI}(\%)$} & \multirow[t]{2}{*}{ EG } & Pre & 13 & $0.08 \pm 12.82$ & \multirow[t]{2}{*}{0.046} \\
\hline & & Post & 13 & $-4.15 \pm 11.72$ & \\
\hline & \multirow[t]{2}{*}{$\mathrm{HG}$} & Pre & 10 & $-2.80 \pm 13.36$ & \multirow[t]{2}{*}{0.205} \\
\hline & & Post & 10 & $-4.80 \pm 12.13$ & \\
\hline & \multirow[t]{2}{*}{$\mathrm{EH}$} & Pre & 14 & $0.15 \pm 8.49$ & \multirow[t]{2}{*}{0.143} \\
\hline & & Post & 14 & $-2.23 \pm 11.81$ & \\
\hline & \multirow[t]{2}{*}{$\mathrm{HH}$} & Pre & 11 & $-5.09 \pm 9.19$ & \multirow[t]{2}{*}{0.062} \\
\hline & & Post & 11 & $-8.45 \pm 9.16$ & \\
\hline \multirow[t]{8}{*}{ Pf (mmHg) } & \multirow[t]{2}{*}{ EG } & Pre & 13 & $26.30 \pm 3.53$ & \multirow[t]{2}{*}{0.918} \\
\hline & & Post & 13 & $26.20 \pm 3.55$ & \\
\hline & \multirow[t]{2}{*}{ HG } & Pre & 10 & $25.40 \pm 3.98$ & \multirow[t]{2}{*}{0.075} \\
\hline & & Post & 10 & $27.30 \pm 5.03$ & \\
\hline & \multirow[t]{2}{*}{$\mathrm{EH}$} & Pre & 14 & $26.54 \pm 4.72$ & \multirow[t]{2}{*}{0.390} \\
\hline & & Post & 14 & $28.00 \pm 5.12$ & \\
\hline & \multirow[t]{2}{*}{$\mathrm{HH}$} & Pre & 11 & $28.20 \pm 3.26$ & 0.729 \\
\hline & & Post & 11 & $28.90 \pm 3.35$ & \\
\hline $\mathrm{Pb}(\mathrm{mmHg})$ & EG & Pre & 13 & $12.90 \pm 3.81$ & 0.243 \\
\hline & & Post & 13 & $11.60 \pm 2.27$ & \\
\hline & HG & Pre & 10 & $12.10 \pm 3.11$ & 0.541 \\
\hline & & Post & 10 & $12.50 \pm 2.99$ & \\
\hline & $\mathrm{EH}$ & Pre & 14 & $12.69 \pm 3.61$ & 0.716 \\
\hline & & Post & 14 & $12.77 \pm 2.55$ & \\
\hline & $\mathrm{HH}$ & Pre & 11 & $13.00 \pm 3.32$ & 0.446 \\
\hline & & Post & 11 & $12.09 \pm 2.81$ & \\
\hline RM (\%) & EG & Pre & 13 & $47.60 \pm 14.39$ & 0.375 \\
\hline & & Post & 13 & $44.10 \pm 8.61$ & \\
\hline & HG & Pre & 10 & $47.70 \pm 14.45$ & 0.775 \\
\hline & & Post & 10 & $47.90 \pm 12.23$ & \\
\hline & $\mathrm{EH}$ & Pre & 14 & $44.15 \pm 8.05$ & 0.645 \\
\hline & & Post & 14 & $44.85 \pm 5.01$ & \\
\hline & $\mathrm{HH}$ & Pre & 11 & $43.55 \pm 9.27$ & 0.230 \\
\hline & & Post & 11 & $41.09 \pm 8.04$ & \\
\hline
\end{tabular}

Table 3. Summary statistics for pre- and post-intervention hemodynamic parameters. ( $\mathrm{BP}=\mathrm{systolic}$ blood pressure; $\mathrm{dBP}=\mathrm{diastolic}$ blood pressure; $\mathrm{PP}=$ pulse pressure; $\mathrm{MAP}=$ mean arterial pressure; $\mathrm{HR}=$ heart rate; $\mathrm{EG}=$ euglycemia; $\mathrm{HG}=$ hyperglycemia; $\mathrm{EH}=$ euglycemic-hyperinsulinemia; $\mathrm{HH}=$ hyperglycemic-hyperinsulinemia; $\mathrm{SD}=$ standard deviation). 


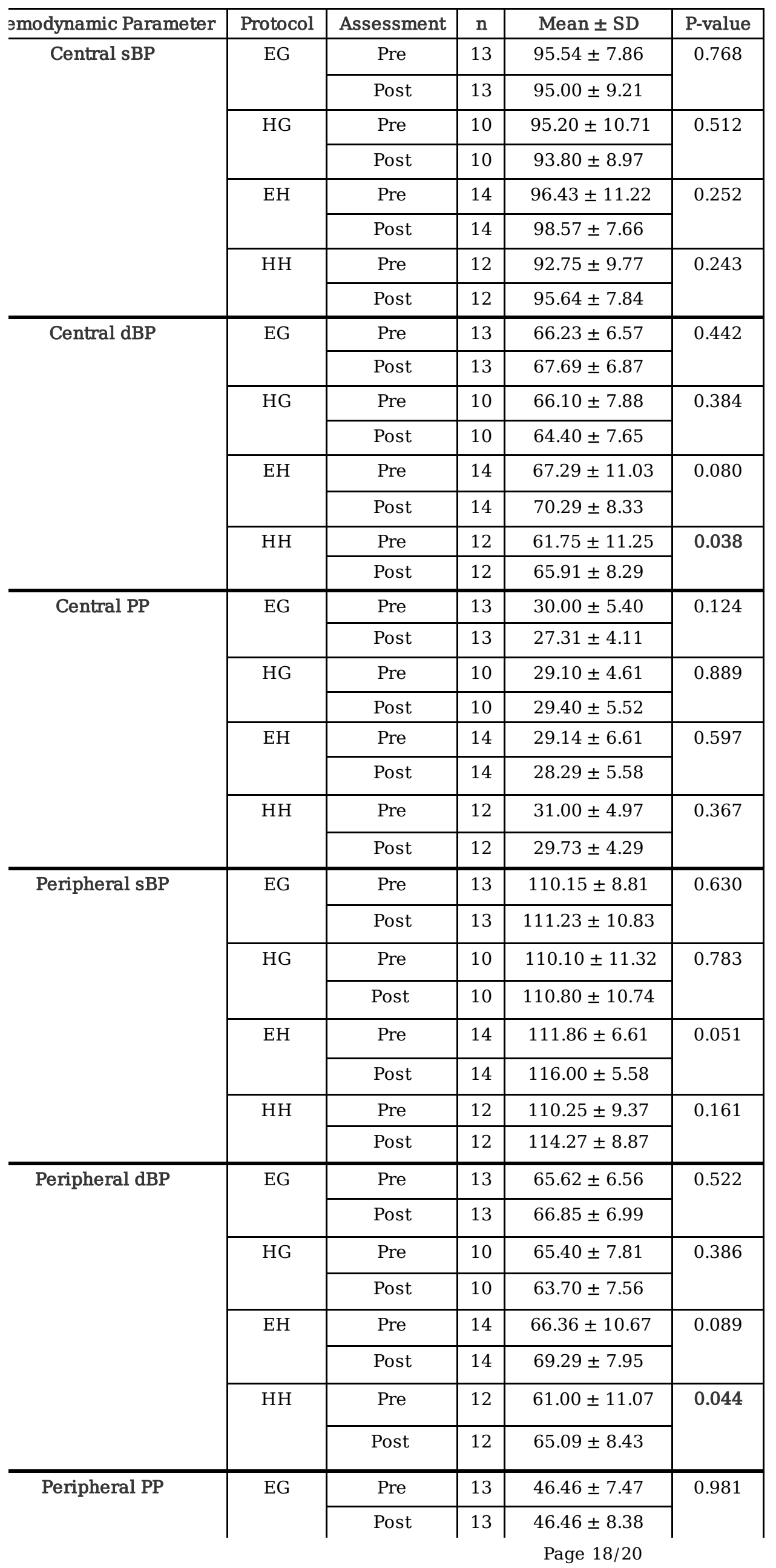




\begin{tabular}{|c|c|c|c|c|}
\hline \multirow{2}{*}{ HG } & Pre & 10 & $44.70 \pm 6.27$ & \multirow{2}{*}{0.379} \\
\cline { 2 - 4 } & Post & 10 & $47.10 \pm 8.27$ & \\
\hline \multirow{2}{*}{ EH } & Pre & 14 & $45.50 \pm 7.86$ & \multirow{2}{*}{0.412} \\
\cline { 2 - 4 } & Post & 14 & $47.43 \pm 9.52$ & \\
\hline \multirow{2}{*}{ HH } & Pre & 12 & $49.25 \pm 5.72$ & \multirow{2}{*}{0.704} \\
\cline { 2 - 4 } & Post & 12 & $49.18 \pm 5.79$ & \\
\hline
\end{tabular}

\begin{tabular}{|c|c|c|c|c|c|}
\hline MAP & EG & Pre & 13 & $79.85 \pm 6.53$ & 0.804 \\
\cline { 3 - 5 } & & Post & 13 & $80.31 \pm 7.39$ & \\
\cline { 2 - 5 } & \multirow{3}{*}{ HG } & Pre & 10 & $78.90 \pm 8.76$ & 0.381 \\
\cline { 3 - 5 } & Post & 10 & $77.30 \pm 8.21$ & \\
\cline { 2 - 5 } & EH & Pre & 14 & $80.50 \pm 10.83$ & \multirow{2}{*}{0.089} \\
\cline { 3 - 5 } & Post & 14 & $83.21 \pm 8.05$ & \\
\cline { 3 - 5 } & HH & Pre & 12 & $75.50 \pm 10.60$ & \multirow{2}{*}{0.033} \\
\cline { 3 - 4 } & Post & 12 & $79.64 \pm 7.98$ & \\
\hline
\end{tabular}

\begin{tabular}{|c|c|c|c|c|c|}
\hline HR & EG & Pre & 13 & $61.92 \pm 10.78$ & \multirow{2}{*}{0.588} \\
\cline { 3 - 5 } & & Post & 13 & $62.67 \pm 9.89$ & \\
\cline { 2 - 5 } & \multirow{2}{*}{ HG } & Pre & 10 & $55.40 \pm 8.22$ & \multirow{2}{*}{0.917} \\
\cline { 3 - 5 } & Post & 10 & $55.10 \pm 6.42$ & \\
\cline { 2 - 5 } & EH & Pre & 14 & $59.64 \pm 9.14$ & \multirow{2}{*}{0.120} \\
\cline { 2 - 4 } & Post & 14 & $61.93 \pm 13.59$ & \\
\cline { 2 - 5 } & HH & Pre & 12 & $58.83 \pm 6.98$ & \multirow{2}{*}{0.009} \\
\cline { 3 - 4 } & Post & 12 & $62.45 \pm 7.17$ & \\
\hline
\end{tabular}

Figures

A

0 min

$240 \mathrm{~min}$

\begin{tabular}{|c|c|c|c|}
\hline \multirow{2}{*}{\multicolumn{2}{|c|}{$-90 \mathrm{~min}$}} & \multicolumn{2}{|c|}{$240 \mathrm{~min}$} \\
\hline & & \multicolumn{2}{|c|}{$\begin{array}{c}\text { Octreotide }(30 \mathrm{ng} / \mathrm{kg} / \mathrm{min})+ \\
\text { Basal Insulin }(0.15 \mathrm{mU} / \mathrm{kg} / \mathrm{min}) \\
\end{array}$} \\
\hline \multicolumn{4}{|c|}{ Euglycemia } \\
\hline $\begin{array}{l}B \\
-90 \min \end{array}$ & \begin{tabular}{|l|}
$\begin{array}{l}\text { Vascular } \\
\text { Studies }\end{array}$ \\
\end{tabular} & & \begin{tabular}{|l|}
$\begin{array}{l}\text { Vascular } \\
\text { Studies }\end{array}$ \\
\end{tabular} \\
\hline$-90 \mathrm{~min}$ & \multicolumn{2}{|c|}{$0 \mathrm{~min}$} & $240 \mathrm{~min}$ \\
\hline \multicolumn{4}{|c|}{ Octreotide $(30 \mathrm{ng} / \mathrm{kg} / \mathrm{min})+$ Basal Insulin $(0.15 \mathrm{mU} / \mathrm{kg} / \mathrm{min})$} \\
\hline \multicolumn{2}{|c|}{ Euglycemia } & \multicolumn{2}{|c|}{ Hyperglycemia ( 200 mg/dL) } \\
\hline & $\begin{array}{l}\text { Vascular } \\
\text { Studies }\end{array}$ & & $\begin{array}{l}\text { Vascular } \\
\text { Studies }\end{array}$ \\
\hline \multicolumn{4}{|l|}{ C } \\
\hline$-90 \mathrm{~min}$ & & $120 \mathrm{~m}$ & $\mathrm{n} \quad 240 \mathrm{~min}$ \\
\hline \multicolumn{3}{|c|}{\begin{tabular}{|c|} 
Basal Insulin $(0.15 \mathrm{mU} / \mathrm{kg} / \mathrm{min})$ \\
Octreotide $(30 \mathrm{ng} / \mathrm{kg} / \mathrm{min})$
\end{tabular}} & $\begin{array}{l}\text { Insulin clamp (1 } \mathrm{mU} / \mathrm{kg} / \mathrm{min}) \\
\text { Continue Octreotide }\end{array}$ \\
\hline \multicolumn{4}{|c|}{ Euglycemia } \\
\hline \multirow[b]{2}{*}{$\begin{array}{l}D \\
-90 \mathrm{~min}\end{array}$} & & \begin{tabular}{l|}
$\begin{array}{l}\text { Vascular } \\
\text { Studies }\end{array}$ \\
\end{tabular} & \begin{tabular}{|l|} 
Vascular \\
Studies
\end{tabular} \\
\hline & in & $120 r$ & $240 \mathrm{~min}$ \\
\hline \multicolumn{3}{|c|}{$\begin{array}{l}\text { Basal Insulin }(0.15 \mathrm{mU} / \mathrm{kg} / \mathrm{min}) \\
\text { Octreotide }(30 \mathrm{ng} / \mathrm{kg} / \mathrm{min})\end{array}$} & $\begin{array}{l}\text { Insulin clamp }(1 \mathrm{mU} / \mathrm{kg} / \mathrm{min}) \\
\text { Continue Octreotide }\end{array}$ \\
\hline \multirow[t]{2}{*}{ Euglycemia } & \multicolumn{3}{|c|}{ Hyperglycemia ( 200 mg/dL) } \\
\hline & & $\begin{array}{l}\text { Vascular } \\
\text { Studies }\end{array}$ & $\begin{array}{l}\text { Vascular } \\
\text { Studies }\end{array}$ \\
\hline
\end{tabular}

\section{Figure 1}

Experimental protocols. $(A=$ euglycemia; $B=$ hyperglycemia; $C=$ euglycemic-hyperinsulinemia; $D=$ hyperglycemic-hyperinsulinemia). 
A

Plasma Glucose
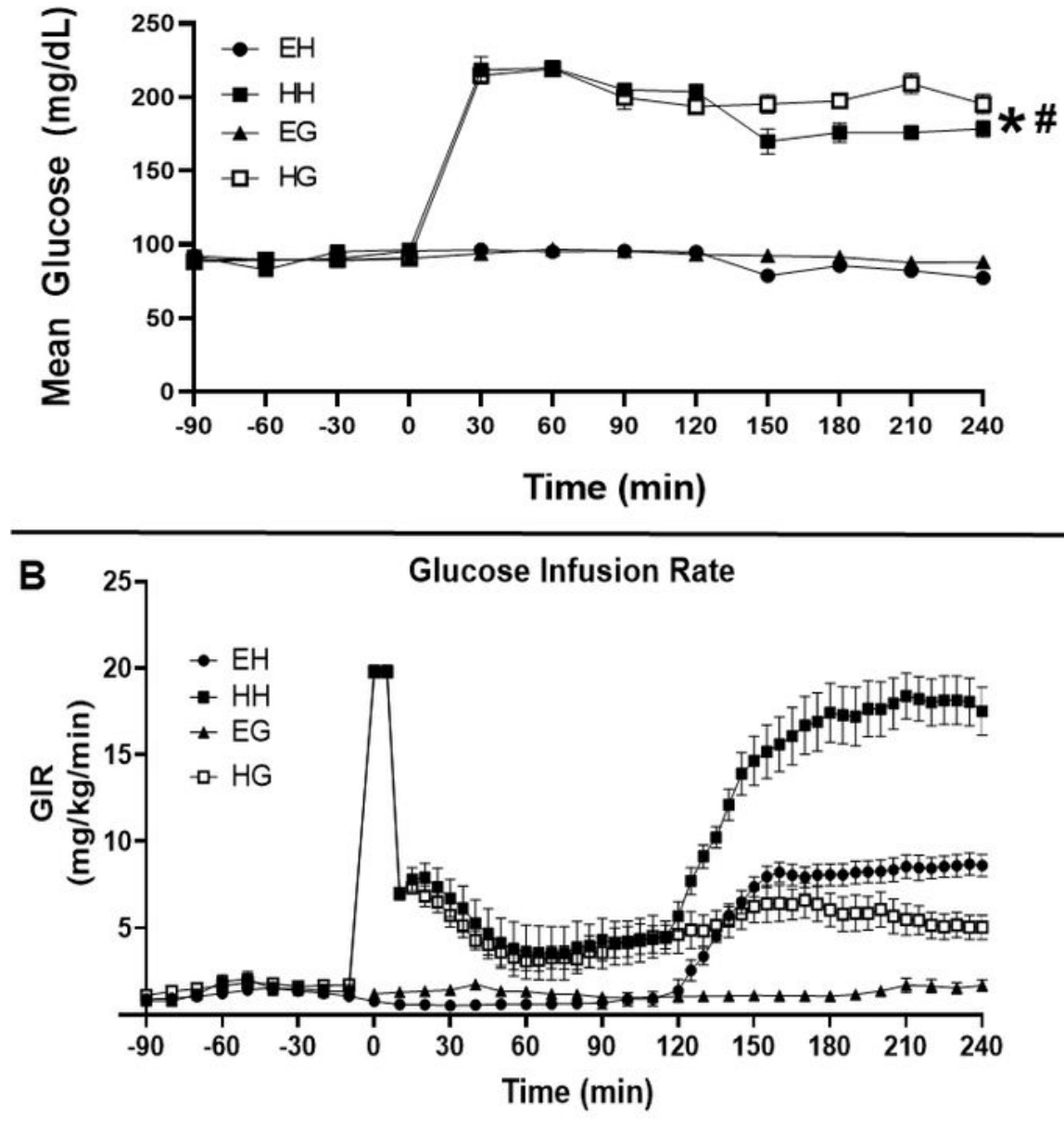

C

\section{Plasma Insulin}

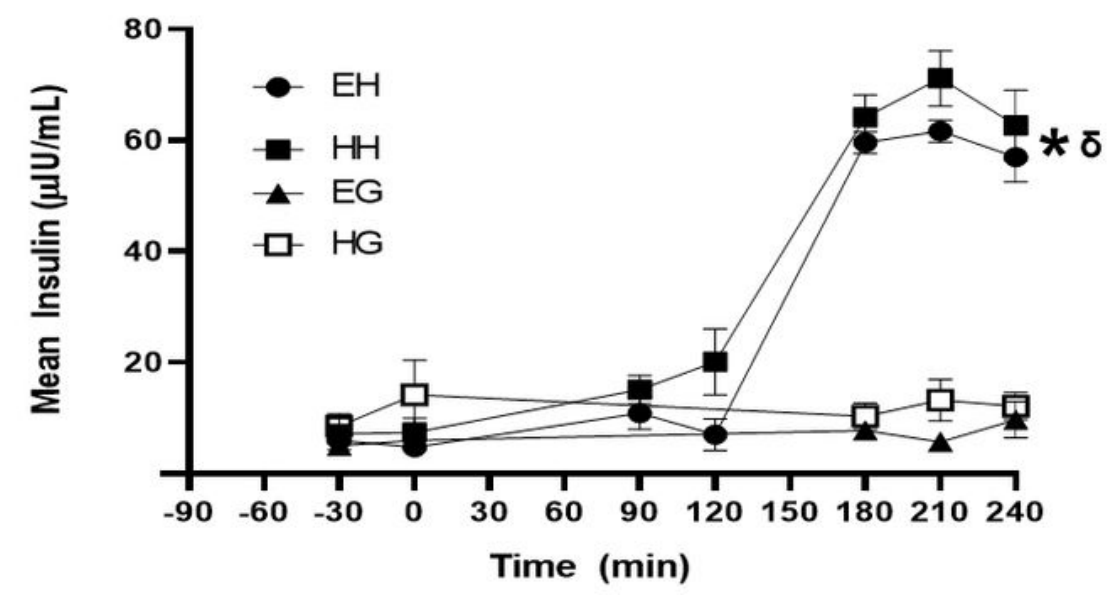

Figure 2

Time course for mean plasma glucose (Panel A), mean glucose infusion rate (Panel B), and mean plasma insulin (Panel $\mathrm{C}$ ) throughout each infusion protocol. (Min= minutes; $\mathrm{GIR}=$ glucose infusion rate; $\mathrm{EG}=$ euglycemia; $\mathrm{HG}=$ hyperglycemia; $\mathrm{EH}=$ euglycemic-hyperinsulinemia; $\mathrm{HH}=$ hyperglycemichyperinsulinemia). ${ }^{*} p<0.001$ when compared to baseline. $\# p<0.01$ when compared to EG or EH. $\delta p<0.001$ when compared to EG or HG. 